\title{
Metrics on Test Function Spaces for Canonical Field Operators
}

\author{
Gerhard C. Hegerfeldt and John R. KLAUder \\ Bell Telephone Laboratories, Murray Hill, New Jersey
}

Received October 19, 1969

\begin{abstract}
In a canonical field theory, the field $\Phi(f)$ and momentum $\pi(g)$ are assumed defined for test functions $f$ and $g$ which are elements of linear vector spaces $\mathscr{V}_{\Phi}$ and $\mathscr{V}_{\pi}$, respectively. Generally, the continuity of the map onto the unitary Weyl operators $U(f)$, $V(g)$ is taken as ray continuity, the barest minimum to recover the field operators as their generators, i.e., $U(f)=e^{i \Phi(f)}, V(g)=e^{i \pi(g)}$. This leaves open the question of whether any wider continuity properties follow and what form they would take. We show that much richer continuity properties do follow in a natural fashion for every cyclic representation of the canonical commutation relations. In particular, we show that the test function space may be taken as a metric space, that the space may be uniquely completed in this topology, and that the map into the unitary Weyl operators is strongly continuous in this topology. The topology induced by this metric is minimal in the sense that it is the weakest vector topology for which the maps $f \rightarrow U(f), g \rightarrow V(g)$ are strongly continuous. An expression for a suitable metric can easily be given in terms of a simple integral over a state on the Weyl operators.
\end{abstract}

\section{Introduction}

By smearing the canonical field operators $\Phi(x)$ and $\pi(x)$ in the usual way with test functions from spaces $\mathscr{V}_{\Phi}$ and $\mathscr{V}_{\pi}$ and then going over to the unitary Weyl operators one arrives at the customary definition of a representation of the canonical commutation relations (CCR) (cf., e.g., [9] or [10]). The only continuity requirement is ray continuity, i.e., in the notation of [1], $U(\lambda f)$ and $V(\lambda g)$ are assumed to be weakly continuous in $\lambda$. One has $V(g) U(f)=\exp \{i(f, g)\} U(f) V(g)$ where $(f, g)$ is the nondegenerate inner product between $\mathscr{V}_{\Phi}$ and $\mathscr{V}_{\pi}$.

The generality and abstractness of this formulation leaves quite open questions about the role of the spaces $\mathscr{V}_{\Phi}$ and $\mathscr{V}_{\pi}$, the relevance of topologies they may carry, the possibility of a wider continuity of the representation than just ray continuity, whether the spaces $\mathscr{V}_{\Phi}$ and $\mathscr{V}_{\pi}$ can be enlarged, etc.

The purpose of this paper is to clarify some of these properties of test function spaces for CCR representations, and specifically to show that there exists a simple and natural metric $d(f, g)$ determined by the representation of the Weyl operators and consistent with the linear vector 
space character, which may be assigned to the space $\mathscr{V}_{\Phi} \times \mathscr{V}_{\pi}$. The test function space may be extended to the closure of this metric space and the mapping of test functions from this metric space onto the Weyl operators is strongly continuous in the metric $d$. Intuitively, there is every reason to expect that the representation of the operators itself should determine - or at least partially determine - the allowed class of smearing functions. In fact, we do find that the representation, through the metric, determines the maximal test function space completely.

In a cyclic representation with cyclic vector $\varphi_{0}$ everything one needs to know about the representation is contained in the oft-studied expectation functional

$$
E(f, g) \equiv\left(\varphi_{0}, U(f) V(g) \varphi_{0}\right) \exp \left\{\frac{i}{2}(f, g)\right\} .
$$

This functional frequently takes a simple form as in the example

$$
E_{r, s}(f, g)=\exp \left\{-\frac{1}{4}\left[(f, f)+(g, g)+s\left(\int f(x) d x\right)^{2}+\operatorname{ir} \int f(x) d x\right]\right\},
$$

which when $r=s=0$ is that characteristic of the elementary Fock representation. Evidently, the functional is well defined for any $g \in L^{2}$ and $f \in L^{2} \cap L^{1}$. We shall shortly indicate that the test function spaces for the representation can be extended consistently to a larger space.

In part, our study was motivated by more abstract topological investigations of Woods [1].

\section{Heuristic Introduction of Metric on Test Function Spaces}

Consider the space $\mathscr{V}_{\Phi}$ and the functional $E(f, 0)=\left(\varphi_{0}, U(f) \varphi_{0}\right)$. The most natural metric to put on $\mathscr{V}_{\Phi}$ is that defined by

$$
d_{1}(f)=\left\|U(f) \varphi_{0}-\varphi_{0}\right\|,
$$

which evidently depends only on $E(f, 0)$. Clearly convergence of $f_{n}$ in $d_{1}$ implies strong convergence of $U\left(f_{n}\right)$ on $\varphi_{0}$, but on what other vectors? Remarkably, as we show in Section 1, this metric leads to the strong convergence of $U\left(f_{n}\right)$ on all of $\mathfrak{H}$. However $d_{1}\left(f_{n}\right)$ is deficient in one important respect for it does not ensure that $U\left(\lambda f_{n}\right)$ also converges for $\lambda \neq 1$. Specific examples exhibiting nonconvergence are easily given. Consider the expectation functional given earlier (with $r=1$ and $s=0$ ) for which

$$
\begin{aligned}
d_{1}^{2}(f) & =2\{1-\operatorname{Re} E(f, 0)\} \\
& =2\left\{1-\exp \left[-\frac{1}{4}(f, f)\right] \cos \left[\frac{1}{4} \int f(x) d x\right]\right\} .
\end{aligned}
$$


One finds that $f_{n}=8 \pi u_{n}(x)$, where (in a one-dimensional configuration space)

$$
\begin{array}{rlrl}
u_{n}(x) & =n^{-1} ; & |x| \leqq n / 2 \\
& =0 ; & & |x|>n / 2,
\end{array}
$$

forms a Cauchy sequence, and that $d_{1}\left(f_{n}\right) \rightarrow 0$. However, $d_{1}\left(\lambda f_{n}\right) \rightarrow 0$ for arbitrary $\lambda$ (e.g., $\lambda=0.5$ ) and the metric would be incompatible with the linear vector character of the space, an aspect we should most like to preserve. To overcome this difficulty one may insist on convergence in the collection of metrics

$$
d_{\lambda}(f) \equiv\left\|U(\lambda f) \varphi_{0}-\varphi_{0}\right\|
$$

for all real $\lambda$. As a weighted sum one might even hope that, for example, convergence in the metric

$$
d^{2}(f)=\int e^{-\lambda^{2}}\left\|U(\lambda f) \varphi_{0}-\varphi_{0}\right\|^{2} d \lambda
$$

accomplishes the same result. That in fact this is the case is the subject of Section 2. There we demonstrate that a suitable metric for $\mathscr{V}_{\Phi} \times \mathscr{V}_{\pi}$ which preserves the linear vector space structure is given by

$$
\begin{aligned}
d^{2}(f, g) & =\int e^{-\lambda^{2}}\left\{\left\|U(\lambda f) \varphi_{0}-\varphi_{0}\right\|^{2}+\left\|V(\lambda g) \varphi_{0}-\varphi_{0}\right\|^{2}\right\} d \lambda \\
& =2 \int e^{-\lambda^{2}}\{2-\operatorname{Re}[E(\lambda f, 0)+E(0, \lambda g)]\} d \lambda .
\end{aligned}
$$

Convergence in this metric, $d\left(f_{n}, g_{n}\right) \rightarrow 0$, implies that $U\left(\lambda f_{n}\right) V\left(\gamma g_{n}\right) \rightarrow \mathbf{1}$ strongly for all $\lambda$ and $\gamma$.

Aspects of completion of the test function space in such a metric and the extension of the representation are discussed in Section 3. It is shown that the Weyl form of the canonical commutation relations is still satisfied but that the extended bilinear form $(f, g)$, although unique, may become degenerate. That is, for some nonzero element $\bar{f}$ in the completion it may happen that $(\bar{f}, g)=0$ for all $g \in \mathscr{V}_{\pi}$. Any such element $U(\bar{f})$ must belong to the center of the algebra generated by $U(f) V(g)$. To illustrate this behavior consider the expectation functional given before, now with $s=1$ and $r=0$. Then it follows (up to a factor $2 \sqrt{\pi}$ ) that

$$
d^{2}(f, g)=1-2\left\{4+(f, f)+\left[\int f(x) d x\right]^{2}\right\}^{-\frac{1}{2}}+1-2\{4+(g, g)\}^{-\frac{1}{2}} .
$$

A Cauchy sequence in this metric is given by

$$
f_{n}(x)=u_{n}(x)
$$

converging to some nonzero element $\bar{f}$ of the completion. It follows (from Schwarz's inequality for example) that

$$
(\bar{f}, g)=0
$$


for all $g \in L^{2}$, and in fact inspection of the representation confirms that $U(\bar{f})$ is a unitary operator lying in the center of the algebra generated by $U(f) V(g)$.

Various topological aspects are discussed in Section 4. In particular, it is shown that the metric $d$ defines the weakest vector topology on $\mathscr{V}_{\Phi}$ and, respectively, $\mathscr{V}_{\pi}$, such that the maps $f \rightarrow U(f), g \rightarrow V(g)$ are strongly continuous. This implies that there are no alternate vector topologies for $\mathscr{V}_{\Phi}, \mathscr{V}_{\pi}$ allowing for a wider extension of the test functions spaces than that already given by the metric.

In Section 5, some measure theoretic properties are discussed. It is shown, for example, that different weight functions also lead to equivalent metrics. Finally an alternate and somewhat stronger proof of the basic theorem of Section 1 is rederived on the basis of a realization of Hilbert space as a direct integral over the space $\mathscr{V}_{\Phi}^{\prime}$ algebraically dual to $\mathscr{V}_{\Phi}$.

We emphasize that extension of CCR representation can always be carried out with the metric $d$, and that this metric provides the maximal extension by vector topologies. As an immediate application of this fact we note that two representations are necessarily inequivalent if the associated metrics are inequivalent for then the completed test function spaces are unequal. Although, of course, the converse is not true this does provide a canonical subclassification of representations.

\section{Representation-Induced Metrics on Test Function Spaces}

In a Schrödinger representation $e^{i \lambda Q}$ has no eigenvectors. Using von Neumann's theorem for CCR and the nondegeneracy of the bilinear form $(f, g)$ one obtains:

Lemma 1.1. Let $U(f, g), f \in \mathscr{V}_{\Phi}, g \in \mathscr{V}_{\pi}$ be a representation of the CCR in some Hilbert space $\mathfrak{H}$, and let $\varphi \in \mathfrak{H}, \varphi \neq 0$. Then

and

$$
d_{1}\left(f-f^{\prime}\right)=\left\|U\left(f-f^{\prime}\right) \varphi-\varphi\right\|=\left\|U(f) \varphi-U\left(f^{\prime}\right) \varphi\right\|
$$

$$
d_{1}\left(g-g^{\prime}\right)=\left\|V\left(g-g^{\prime}\right) \varphi-\varphi\right\|
$$

define a metric on $\mathscr{V}_{\Phi}$ and, respectively, $\mathscr{V}_{\pi}$.

We note the following simple consequences:

Lemma 1.2. Let $\mathfrak{H}_{\varphi}$ be the closed subspace generated by all vectors of the form $U(f) \varphi, f \in \mathscr{V}_{\Phi}$. Then $\left\|U\left(f_{n}\right) \varphi-\varphi\right\| \rightarrow 0$ implies $\left\|U\left(f_{n}\right) \psi-\psi\right\|$ $\rightarrow 0$ for all $\psi \in \mathfrak{H}_{\varphi}$.

Proof. First consider $U(f) \varphi$. Then

$$
\left\|\left(U\left(f_{n}\right)-\mathbf{1}\right) U(f) \varphi\right\|=\left\|U(f)\left(U\left(f_{n}\right)-\mathbf{1}\right) \varphi\right\|=\left\|U\left(f_{n}\right) \varphi-\varphi\right\| \rightarrow 0 .
$$


Hence $\left\|U\left(f_{n}\right) \psi_{M}-\psi_{M}\right\| \rightarrow 0$ for all finite linear combinations

$$
\psi_{M}=\sum_{1}^{M} \alpha_{i} U\left(f_{i}^{\prime}\right) \varphi
$$

This set is dense in $\mathfrak{h}_{\varphi}$. But if $\left\|U\left(f_{n}\right) \psi_{M}-\psi_{M}\right\| \rightarrow 0$ for a dense set of vectors, then $U\left(f_{n}\right) \rightarrow \mathbf{1}$ strongly on $\mathfrak{H}_{0}$ since $\left\|U\left(f_{n}\right) \psi-\psi\right\| \leqq \| U\left(f_{n}\right) \psi$ $-U\left(f_{n}\right) \psi_{M}\|+\| U\left(f_{n}\right) \psi_{M}-\psi_{M}\|+\| \psi_{M}-\psi \|$. q.e.d.

The previous lemma can be used to show that for a separable space or under even more general conditions one can always find a vector $\varphi$ such that $\left\|U\left(f_{n}-f_{0}\right) \varphi-\varphi\right\| \rightarrow 0$ implies strong convergence. Both the previous and the next lemma are independent of whether $V(g)$ exists or not. Hence they are true for any Abelian group of unitary operators.

Lemma 1.3. Let $\mathfrak{H}$ be separable [or, more generally, let $\mathfrak{H}$ decompose into a countable direct sum of subspaces $\mathfrak{H}_{i}$ which are cyclic with respect to $U(f)]$. Then there exists a vector $\varphi_{0} \in \mathfrak{H}$ such that $\left\|U\left(f_{n}\right) \varphi_{0}-\varphi_{0}\right\| \rightarrow 0$ implies $U\left(f_{n}\right) \rightarrow \mathbf{1}$ strongly.

Proof. First note that if $\mathfrak{H}$ is separable then it decomposes into $\mathfrak{H}=\bigoplus_{i} \mathfrak{H}_{i}$ where each $\mathfrak{H}_{i}$ is cyclic for $U(f), f \in \mathscr{V}_{\Phi}$ (cf., e.g., the construction in [2] for a one-parameter group). Let the unit vectors $\varphi_{i}$ be cyclic in $\mathfrak{H}_{i}, i=1,2, \ldots$, and define

Then

$$
\varphi_{0}=\sum_{i} \oplus \frac{1}{2^{i / 2}} \varphi_{i}
$$

$$
\left\|U\left(f_{n}\right) \varphi_{0}-\varphi_{0}\right\|^{2}=\sum_{i} \frac{1}{2^{i}}\left\|U\left(f_{n}\right) \varphi_{i}-\varphi_{i}\right\|^{2},
$$

and convergence to 0 implies convergence to 0 for each term in the sum. But then, by Lemma 1.2, $\left\|U\left(f_{n}\right) \psi_{i}-\psi_{i}\right\| \rightarrow 0$ for each $\psi_{i} \in \mathfrak{S}_{i}, i=1,2, \ldots$, and therefore $U\left(f_{n}\right) \rightarrow \mathbf{1}$ on a dense set of vectors. Hence $U\left(f_{n}\right) \rightarrow \mathbf{1}$ strongly. q.e.d.

The above lemmas exploit the commutativity of $U\left(f_{n}\right)$ and $U(f)$. If $\varphi_{0}$ is cyclic for $U(f, g)=U(f) V(g)$, then one has

$$
\begin{aligned}
& \left\|\left(U\left(f_{n}\right)-1\right) U(f) V(g) \varphi_{0}\right\|=\left\|U(f) V(g)\left\{e^{-i\left(f_{n}, g\right)} U\left(f_{n}\right)-1\right\} \varphi_{0}\right\| \\
& \leqq\left\|U\left(f_{n}\right) \varphi_{0}-\varphi_{0}\right\|+\left|e^{-i\left(f_{n}, g\right)}-1\right|\left\|\varphi_{0}\right\| \text {. }
\end{aligned}
$$

Hence, if $e^{i\left(f_{n}, g\right)} \rightarrow 1$ for $n \rightarrow \infty$ for all $g \in \mathscr{V}_{\pi}$, then $\left\|U\left(f_{n}\right) \psi-\psi\right\| \rightarrow 0$ for a dense set of vectors.

On the other hand, one sees from

$$
\left\|\left(U\left(f_{n}\right)-1\right) U(f, g) \varphi\right\| \geqq\left|\left\|U\left(f_{n}\right) \varphi_{0}-\varphi_{0}\right\|-\right| e^{-i\left(f_{n}, g\right)}-1\left|\left\|\varphi_{0}\right\|\right|
$$


that $e^{i\left(f_{n}, g\right)} \rightarrow 1$ is also necessary for strong convergence. Note that from $e^{i \lambda\left(f_{n}, g\right)} \rightarrow 1$ for all real $\lambda$ in some interval one obtains as in [1] by integration

$$
\lim _{n}\left(f_{n}, g\right)=0 \text {. }
$$

Similar considerations apply to $V\left(g_{n}\right)$. Thus one has the following:

Lemma 1.4. Let $\varphi_{0}$ be cyclic for $U(f) V(g), f \in \mathscr{V}_{\Phi}, g \in \mathscr{V}_{\pi}$. Then a) $U\left(f_{n}\right) \rightarrow 1$ strongly if and only if $\left\|U\left(f_{n}\right) \varphi_{0}-\varphi_{0}\right\| \rightarrow 0$ and $\left(f_{n}, g\right) \rightarrow 0$ for each $g \in \mathscr{V}_{\pi}$. b) $V\left(g_{n}\right) \rightarrow \mathbf{1}$ strongly if and only if $\left\|V\left(g_{n}\right) \varphi_{0}-\varphi_{0}\right\| \rightarrow 0$ and $\left(f, g_{n}\right) \rightarrow 0$ for each $f \in \mathscr{V}_{\Phi}$.

Comparing Lemmas 1.2 and 1.4 one sees that if $\varphi_{0}$ is not only cyclic for $U(f, g)$ but also cyclic for $U(f)$ alone, then $\left\|U\left(f_{n}\right) \varphi_{0}-\varphi_{0}\right\| \rightarrow 0$ already implies $\left(f_{n}, g\right) \rightarrow 0$ for each $g \in \mathscr{V}_{\pi}$. One therefore wonders if the condition $\left(f_{n}, g\right) \rightarrow 0$ is already a consequence of $\left\|U\left(f_{n}\right) \varphi_{0}-\varphi_{0}\right\| \rightarrow 0$. The following theorem shows that this is even true for any vector $\varphi$ for any representation, quite a remarkable property.

Theorem 1.5. Let $U(f), V(g)$ be a representation of the CCR in some Hilbert space $\mathfrak{G}$, with $f \in \mathscr{V}_{\Phi}, g \in \mathscr{V}_{\pi}$. Let $\varphi \in \mathfrak{H}, \varphi \neq 0$, and let $f_{n} \in \mathscr{V}_{\Phi}$, $n=1,2, \ldots$. If

$$
\left\|U\left(f_{n}\right) \varphi-\varphi\right\| \rightarrow 0
$$

then

$$
\left(f_{n}, g\right) \rightarrow 0 \text { for all } g \in \mathscr{V}_{\pi} .
$$

If $\left\|V\left(g_{n}\right) \varphi-\varphi\right\| \rightarrow 0$, then $\left(f, g_{n}\right) \rightarrow 0$ for all $f \in \mathscr{V}_{\Phi}$.

Proof ${ }^{1}$. By Lemma 1.2, $U\left(f_{n}\right) \rightarrow \mathbf{1}$ strongly on $\mathfrak{H}_{\varphi}=\overline{\{U(f) \varphi\}}$. Consider a $g \in \mathscr{V}_{\pi}$ and a real number $\lambda$. Decompose $V(\lambda g) \varphi$ into its orthogonal components $\chi_{\lambda} \in \mathfrak{H}_{\varphi}$ and $\chi_{\lambda}^{\prime} \in \mathfrak{H}_{\varphi}^{\prime}$, the subspace orthogonal to $\mathfrak{H}_{\varphi}$,

$$
V(\lambda g) \varphi=\chi_{\lambda}+\chi_{\lambda}^{\prime} \text {. }
$$

Note that $\mathfrak{H}_{\varphi}^{\prime}$ is also invariant under $U(f), f \in \mathscr{V}_{\Phi}$. Since $V(\lambda g)$ is continuous in $\lambda$, there is a $\delta>0$ such that $\chi_{\lambda} \neq 0$ for $|\lambda| \leqq \delta$. Now,

$$
\begin{aligned}
& \|\left(U\left(f_{n}\right)-1\right) V(-\lambda g) V(\lambda g) \varphi\left\|^{2}=\right\|\left(e^{i \lambda\left(f_{n}, g\right)} U\left(f_{n}\right)-1\right)\left(\chi_{\lambda}+\chi_{\lambda}^{\prime}\right) \|^{2} \\
&=\left\|\left(e^{i \lambda\left(f_{n}, g\right)} U\left(f_{n}\right)-1\right) \chi_{\lambda}\right\|^{2}+\left\|\left(e^{i \lambda\left(f_{n}, g\right)} U\left(f_{n}\right)-1\right) \chi_{\lambda}^{\prime}\right\|^{2} \\
& \geqq|| e^{i \lambda\left(f_{n}, g\right)}-1\left|\cdot\left\|\chi_{\lambda}\right\|-\left\|\left(U\left(f_{n}\right)-1\right) \chi_{\lambda}\right\|\right|^{2} .
\end{aligned}
$$

Since $\chi_{\lambda} \in \mathfrak{H}_{\varphi}$, the last term tends to zero for $n \rightarrow \infty$. Hence

$$
e^{i \lambda\left(f_{n}, g\right)} \rightarrow 1 \text { for }|\lambda| \leqq \delta .
$$

Then Eq. (1.2) implies $\left(f_{n}, g\right) \rightarrow 0$.

${ }^{1}$ We owe this very elegant and simple version to L. Streit. The connection of this theorem with measure-theoretic properties of the CCR will be discussed in Section 5. 
Replacing $U\left(f_{n}\right)$ by $V\left(g_{n}\right)$ and $V(\lambda g)$ by $U(-\lambda f)$ in the above argument one obtains $\left(f, g_{n}\right) \rightarrow 0$. q.e.d.

For finitely many degrees of freedom $\mathscr{V}_{\Phi}$ and $\mathscr{V}_{\pi}$ are $N$-dimensional vector spaces, and $\left(f_{n}, g\right) \rightarrow 0$ for all $g \in \mathscr{V}_{\pi}$ means that all components of $f_{n}$ in any basis tend to 0 . Thus the above theorem shows as a corollary that in this case the metric $\|U(f) \varphi-\varphi\|$ is just equivalent to a Euclidean metric on $\mathscr{V}_{\Phi}$, a result already obtained by Woods [1] by a more complicated technique.

Combining the above theorem with Lemma 1.4, one finds the following:

Corollary 1.6. Let $\varphi_{0}$ be a cyclic vector for a representation $U(f)$, $V(g)$ of the CCR, with $f \in \mathscr{V}_{\Phi}, g \in \mathscr{V}_{\pi}$. Then $\left\|U\left(f_{n}\right) \varphi_{0}-\varphi_{0}\right\| \rightarrow 0$ implies $U\left(f_{n}\right) \rightarrow \mathbf{1}$ strongly; and $\left\|V\left(g_{n}\right) \varphi_{0}-\varphi_{0}\right\| \rightarrow 0$ implies $V\left(g_{n}\right) \rightarrow \mathbf{1}$ strongly.

Each representation of the CCR always decomposes into a (possibly uncountable) direct sum of cyclic representations. If the sum is countable one can proceed as in the proof of Lemma 1.3 to obtain the following generalization:

Corollary 1.7. Let $U(f), V(g)$ with $f \in \mathscr{V}_{\Phi}, g \in \mathscr{V}_{\pi}$ be a representation in a separable $\mathfrak{H}$ (or, more generally, let it decompose into a countable direct sum of cyclic representations). Then there is a vector $\varphi_{0} \in \mathfrak{H}$ such that $\left\|U\left(f_{n}\right) \varphi_{0}-\varphi_{0}\right\| \rightarrow 0$ implies $U\left(f_{n}\right) \rightarrow \mathbf{1}$ strongly, and $\left\|V\left(g_{n}\right) \varphi_{0}-\varphi_{0}\right\| \rightarrow 0$ implies $V\left(g_{n}\right) \rightarrow 1$ strongly.

Thus we see that the metrics $d_{1}(f)=\left\|U(f) \varphi_{0}-\varphi_{0}\right\|$ on $\mathscr{V}_{\Phi}$ and $d_{1}(g)=\left\|V(g) \varphi_{0}-\varphi_{0}\right\|$ on $\mathscr{V}_{\pi}$ with $\varphi_{0}$ cyclic (or $\varphi_{0}$ as in Corollary 1.7) make the maps $f \rightarrow U(f), g \rightarrow V(g)$ strongly continuous.

\section{Metrics Compatible with the Vector Space Structure}

As already pointed out in the introduction $f_{n} \rightarrow f\left(d_{1}\right)$ does not necessarily imply $\lambda f_{n} \rightarrow \lambda f\left(d_{1}\right)$. But this is a property one would demand from a metric on a vector space. In addition to $d_{1}\left(f_{n}\right)$ one should therefore also take into account the metrics

$$
d_{\lambda}\left(f_{n}\right) \equiv d_{1}\left(\lambda f_{n}\right)=\left\|U\left(\lambda f_{n}\right) \varphi-\varphi\right\| \text {. }
$$

Note that $d_{\lambda}$ satisfies $d_{\lambda}(f)=d_{\lambda}(-f)$. The metrics $d_{\lambda}$ are uncountably many. In order to construct a new metric with them one might consider

$$
d\left(f_{n}\right)^{2}=\int_{-\infty}^{\infty} d \lambda e^{-\lambda^{2}}\left\|U\left(\lambda f_{n}\right) \varphi-\varphi\right\|^{2},
$$

or a similar expression with some other weight function. One might also replace the integration limits by $a, b$ with $a<b$. As in the theory of $L^{p}$-spaces one finds: 
Lemma 2.1. $\left\{\int d \sigma(\lambda) d_{\lambda}(f)^{2}\right\}^{\frac{1}{2}}$ defines a metric.

In order that the metric $d$ in (2.2) serves its purpose one needs that $d\left(f_{n}\right) \rightarrow 0$ implies $d_{\lambda}\left(f_{n}\right) \rightarrow 0$ for every $\lambda$. This seems to be quite a strong requirement, for from (2.2) one cannot even conclude that $d_{\lambda}\left(f_{n}\right) \rightarrow 0$ for almost all $\lambda$. It only follows that $d_{\lambda}\left(f_{n}\right), n=1,2, \ldots$, considered as a sequence of functions of $\lambda$, converges to 0 in measure. Therefore the following result may be somewhat surprising.

Lemma 2.2. $d\left(f_{n}\right) \rightarrow 0$ if and only if $d_{\lambda}\left(f_{n}\right) \rightarrow 0$ for each real $\lambda$.

Proof. Let $d\left(f_{n}\right) \rightarrow 0$. For each $n$, one can write, by Stone's theorem,

$$
U\left(\lambda f_{n}\right)=\int_{-\infty}^{\infty} e^{i \lambda x} d E_{x}^{(n)} .
$$

Thus

$$
\begin{aligned}
\left\|U\left(\lambda f_{n}\right) \varphi-\varphi\right\|^{2} & =2(\varphi, \varphi)-\left(\varphi,\left\{U\left(\lambda f_{n}\right)+U\left(-\lambda f_{n}\right)\right\} \varphi\right) \\
& =\int\left|e^{i \lambda x}-1\right|^{2} d\left(\varphi, E_{x}^{(n)} \varphi\right) .
\end{aligned}
$$

Hence, putting $d\left(\varphi, E_{x}^{(n)} \varphi\right) \equiv d \mu_{n}(x)$,

$$
d\left(f_{n}\right)^{2}=\int d \lambda e^{-\lambda^{2}} \int d \mu_{n}(x)\left|e^{i \lambda x}-1\right|^{2},
$$

and for any $1>\eta>0$ one obtains similarly as in [1] by an allowed change of the order of integration

$$
\begin{aligned}
d\left(f_{n}\right)^{2} & \geqq \frac{1}{e} \int_{0}^{1} d \lambda \int_{|x|>\eta}\left|e^{i \lambda x}-1\right|^{2} d \mu_{n}(x) \\
& \geqq \frac{1}{e} \cdot 2\left(1-\eta^{-1} \sin \eta\right) \int_{|x|>\eta} d \mu_{n}(x) .
\end{aligned}
$$

Hence for each $0<\eta<1$

$$
\lim _{n \rightarrow \infty} \int_{|x|>\eta} d \mu_{n}(x)=0 .
$$

Now let $\lambda_{0}$ be any real number. For any $\varepsilon>0$ there is $0<\eta<1$ such that

$$
\left|e^{i \lambda_{0} x}-1\right|^{2}<\varepsilon / 2\|\varphi\|^{2} \text { for all }|x| \leqq \eta .
$$

Thus

$$
\begin{aligned}
d_{\lambda_{0}}\left(f_{n}\right)^{2}= & \int\left|e^{i \lambda_{0} x}-1\right|^{2} d \mu_{n}(x) \\
& \leqq \frac{\varepsilon}{2\|\varphi\|^{2}} \cdot\|\varphi\|^{2}+4 \int_{|x|>\eta} d \mu_{n}(x) \\
& \leqq \varepsilon / 2+\varepsilon / 2
\end{aligned}
$$

for $n$ greater than some $N(\varepsilon)$. Hence $d_{\lambda_{0}}\left(f_{n}\right) \rightarrow 0$. 
The converse part of the lemma follows immediately from Lebesgue's bounded convergence theorem. q.e.d.

Corollary 2.3. If $d\left(f_{n}\right) \rightarrow 0$, then $d_{\lambda}\left(f_{n}\right) \rightarrow 0$ uniformly in $\lambda$ on every bounded interval.

Proof. This follows immediately from Eq. (2.8). q.e.d.

Note that the preceding lemma holds for arbitrary Hilbert spaces, not only for separable ones, and that one can take any vectors $\varphi \in \mathfrak{H}$. As an immediate corollary one also obtains convergence of $\lambda f_{n}$ in the metric $d$.

Corollary 2.4. If $f_{n} \rightarrow f$ in the metric $d$, then also $\lambda f_{n} \rightarrow \lambda f$ in $d$.

Proof. Put $\lambda\left(f_{n}-f\right)=f_{n}^{\prime}$. Then $d_{\lambda^{\prime}}\left(f_{n}^{\prime}\right) \rightarrow 0$ for each real $\lambda^{\prime}$. Hence $d\left(f_{n}^{\prime}\right) \rightarrow 0$. q.e.d.

Thus one sees that the metric $d$ has just the property looked for. Convergence of $f_{n}$ implies convergence of $\lambda f_{n}$, and if one chooses for $\varphi$ a cyclic vector or the $\varphi_{0}$ of Corollary 1.7, then $f_{n} \rightarrow f(d)$ implies strong convergence $U\left(\lambda f_{n}\right) \rightarrow U(\lambda f)$ for each $\lambda$.

But there is one further property one would like a metric on a vector space to fulfill, i.e., if $\lambda_{n} \rightarrow \lambda_{0}$ and $f_{n} \rightarrow f_{0}$ one would like to have $\lambda_{n} f_{n} \rightarrow \lambda_{0} f_{0}$. The following lemma shows that $d$ also fulfills this.

Lemma 2.5. Let $\lambda_{n} \rightarrow \lambda_{0}$ and $f_{n} \rightarrow f_{0}(d)$. Then $\lambda_{n} f_{n} \rightarrow \lambda_{0} f_{0}(d)$.

Proof. One has

$$
d\left(\lambda_{n} f_{n}-\lambda_{0} f_{0}\right) \leqq d\left(\lambda_{n} f_{n}-\lambda_{n} f_{0}\right)+d\left(\left(\lambda_{n}-\lambda_{0}\right) f_{0}\right) .
$$

For $n \rightarrow \infty,\left\|U\left(\lambda\left(\lambda_{n}-\lambda_{0}\right) f_{0}\right) \varphi-\varphi\right\| \rightarrow 0$ by the ray continuity of the representation. Hence $d\left(\left(\lambda_{n}-\lambda_{0}\right) f_{0}\right) \rightarrow 0$, by Lemma 2.2. So it suffices to show that $d\left(\lambda_{n}\left(f_{n}-f_{0}\right)\right) \rightarrow 0$, and one can assume $f_{0}=0$. By Lemma 2.2, $d\left(f_{n}\right) \rightarrow 0$ if and only if $d_{\lambda}\left(f_{n}\right) \rightarrow 0$. Putting $\|\varphi\|=1$, Eq. (2.4) shows that this is the case if and only if

$$
\operatorname{Re}\left(\varphi, U\left(\lambda f_{n}\right) \varphi\right) \rightarrow 1 .
$$

Since $\left|\left(\varphi, U\left(\lambda f_{n}\right) \varphi\right)\right| \leqq\|\varphi\|^{2}=1, d_{\lambda}\left(f_{n}\right) \rightarrow 0$ if and only if

$$
K_{n}(\lambda) \equiv\left(\varphi, U\left(\lambda f_{n}\right) \varphi\right) \rightarrow\|\varphi\|^{2}=1 .
$$

By Stone's theorem, Eq. (2.3),

$$
K_{n}(\lambda)=\int e^{i \lambda x} d\left(\varphi, E_{x}^{(n)} \varphi\right) \equiv \int e^{i \lambda x} d \mu_{n}(x) .
$$

Since $\int d \mu_{n}(x)=\|\varphi\|^{2}=1, K_{n}(\lambda)$ is the characteristic function of a probability measure, and $K_{n}(\lambda) \rightarrow K_{0}(\lambda) \equiv 1$ for each $\lambda$. Now, if $\lambda_{n} \rightarrow \lambda_{0}$, then, by [3], $K_{n}\left(\lambda_{n}\right) \rightarrow K_{0}\left(\lambda_{0}\right)=1$. Replacing $\lambda_{n}$ by $\lambda^{\prime} \lambda_{n}$ and $\lambda_{0}$ by $\lambda^{\prime} \lambda_{0}$, one has $K_{n}\left(\lambda^{\prime} \lambda_{n}\right) \rightarrow K_{0}\left(\lambda^{\prime} \lambda_{0}\right)=1$ for each $\lambda^{\prime}$. Hence $\left(\varphi, U\left(\lambda^{\prime} \lambda_{n} f_{n}\right) \varphi\right) \rightarrow 1$ for each $\lambda^{\prime}$, i.e., $d_{\lambda^{\prime}}\left(\lambda_{n} f_{n}\right) \rightarrow 0$. q.e.d. 
It should be remarked that the form of the weight function in forming $d(f)$ is inessential. It is clear, for instance, that instead of $d(f)$ one could also take

$$
\tilde{d}(f)=\left\{\int_{0}^{1} d \lambda\|U(\lambda f) \varphi-\varphi\|^{2}\right\}^{\frac{1}{2}} .
$$

Then Lemma 2.2 holds and hence also Corollary 2.4 and Lemma 2.5 which are based on Lemma 2.2. In Section 5 the most general weight function and metric will be determined.

The results of Sections 1 and 2 can be combined to yield the following theorem. Then

Theorem 2.6. Let $\varphi_{0}$ be a cyclic vector for $U(f), V(g), f \in \mathscr{V}_{\Phi}, g \in \mathscr{V}_{\pi}$.

$$
d(f)^{2}=\int d \lambda e^{-\lambda^{2}}\left\|U(\lambda f) \varphi_{0}-\varphi_{0}\right\|^{2}
$$

defines a metric on $\mathscr{V}_{\Phi}$ with the following properties. a) $f_{n} \rightarrow f(d)$ if and only if $U\left(\lambda f_{n}\right) \rightarrow U(\lambda f)$ strongly for all $\lambda$. b) If $f_{n} \rightarrow f_{0}(d)$ and $\lambda_{n} \rightarrow \lambda_{0}$ then $\lambda_{n} f_{n} \rightarrow \lambda_{0} f_{0}(d)$ and $U\left(\lambda_{n} f_{n}\right) \rightarrow U\left(\lambda_{0} f_{0}\right)$ strongly. Analogous results hold for $U(f)$ replaced by $V(g)$.

To express $d(f)$ in terms of the expectation functional $E(f, g)$, one can write

$$
d(f)^{2}=2 \int d \lambda e^{-\lambda^{2}} \operatorname{Re}\{1-E(\lambda f, 0)\},
$$

and for the metric on $\mathscr{V}_{\pi}$ one replaces $E(f, 0)$ by $E(0, g)$. Thus Theorem 2.6 can be rephrased in terms of the expectation functional which determines the representation uniquely up to unitary equivalence.

It is clear that, instead of considering metrics on $\mathscr{V}_{\Phi}$ and $\mathscr{V}_{\pi}$ separately, one can also combine them to a common metric on $\mathscr{V}_{\Phi} \times \mathscr{V}_{\pi}$ by defining the distance of a couple $\{f, g\}$ from $\{0,0\}$ by the expression $d(f, g)$ of the introduction.

\section{Extension of Representations}

A straightforward application of the above results yields an extension of the representation to larger test function spaces, the completions $\overline{\mathscr{V}}_{\Phi}$ and $\overline{\mathscr{V}}_{\pi}$. Since for metrics this can be done without invoking deeper topological theorems we outline the simple procedure.

The completions $\overline{\mathscr{V}}_{\Phi}$ and $\overline{\mathscr{V}}_{\pi}$ are constructed in a well-known manner by considering equivalence classes of Cauchy sequences. One defines $d(\bar{f})$ where $\bar{f}=\lim f_{n}$ by $\lim d\left(f_{n}\right)$. This limit exists since, by the triangle inequality, $d\left(f_{n}-f_{m}\right) \geqq\left|d\left(f_{n}\right)-d\left(f_{m}\right)\right|$. The same construction applies of course also to $\mathscr{V}_{\pi}$. 
In order to extend the bilinear form $(f, g)$ from $\mathscr{V}_{\Phi} \times \mathscr{V}_{\pi}$ to $\overline{\mathscr{V}}_{\Phi} \times \overline{\mathscr{V}}_{\pi}$, put

$$
(\bar{f}, \bar{g})=\lim _{n}\left(f_{n}, g_{n}\right) .
$$

The limit exists since $\left(f_{n}, g_{n}\right)$ is a Cauchy sequence. This follows, as in Eq. (1.3), by considering $\left\|U\left(\lambda f_{n}\right) V\left(g_{n}\right) \varphi-U\left(\lambda f_{m}\right) V\left(g_{m}\right) \varphi\right\|$. Uniqueness of definition and linearity follow in a straightforward way from Theorems 1.5 and 2.6.

In order to extend the representation, one can invoke a general theorem on mappings of topological groups [4]. In the present simple situation one can also proceed directly as follows.

Let $f_{n} \rightarrow \bar{f}$. Then $U\left(\lambda\left(f_{n}-f_{m}\right)\right) \rightarrow \mathbf{1}$ strongly so that $U\left(\lambda f_{n}\right)$ is a Cauchy sequence in the strong operator topology for each $\lambda$. Since the space $B_{1}(\mathfrak{H})$ of operators $A$ on $\mathfrak{H}$ with $\|A\| \leqq 1$ is complete [5] in the strong operator topology, $U\left(\lambda f_{n}\right)$ converges strongly to some operator $A_{\lambda}$. From

$$
\begin{aligned}
U\left(\left(\lambda_{1}+\lambda_{2}\right) f_{n}\right)-A_{\lambda_{1}} A_{\lambda_{2}}= & U\left(\lambda_{1} f_{n}\right)\left(U\left(\lambda_{2} f_{n}\right)-A_{\lambda_{2}}\right) \\
& +\left(U\left(\lambda_{1} f_{n}\right)-A_{\lambda_{1}}\right) A_{\lambda_{2}}
\end{aligned}
$$

it follows that $U\left(\left(\lambda_{1}+\lambda_{2}\right) f_{n}\right)=U\left(\lambda_{1} f_{n}\right) U\left(\lambda_{2} f_{n}\right) \rightarrow A_{\lambda_{1}+\lambda_{2}}=A_{\lambda_{1}} A_{\lambda_{2}}$. From $U\left(\lambda f_{n}\right)^{*}=U\left(-\lambda f_{n}\right)$ one sees that $A_{\lambda}^{*}=A_{-\lambda}$ and $A_{0}=1$. Furthermore, for any $\psi \in \mathfrak{H},\left(\psi,\left[U\left(\lambda f_{n}\right)-U\left(\lambda f_{m}\right)\right] \psi\right) \rightarrow 0$ uniformly in $\lambda$ on every bounded interval, by Corollary 2.3. Hence $\left(\psi, A_{\lambda} \psi\right)=\lim \left(\psi, U\left(\lambda f_{n}\right) \psi\right)$ is continuous in $\lambda$, and $A_{\lambda}$ is a continuous one-parameter unitary group. Define

$$
U(\lambda \bar{f})=A_{\lambda} .
$$

By Theorem 2.6, $U(\lambda \bar{f})$ cannot equal 1 for all $\lambda$ if $\bar{f} \neq 0$. Since any other Cauchy sequence $f_{n}^{\prime} \rightarrow \bar{f}$ differs from $f_{n}$ by a null sequence, it is immediate that $U\left(\lambda f_{n}^{\prime}\right)$ converges also to $A_{\lambda}$ so that the definition of $U(\lambda \bar{f})$ depends only on $\bar{f}$.

The same construction can be carried through for $V(\bar{g})$. As in Eq. (3.2) one has

$$
\begin{aligned}
U(\bar{f}) V(\bar{g}) & =\operatorname{str} . \lim U\left(f_{n}\right) V\left(g_{n}\right)=\operatorname{str} . \lim e^{-i\left(f_{n}, g_{n}\right)} V\left(g_{n}\right) U\left(f_{n}\right) \\
& =e^{-i(\bar{f}, \bar{g})} V(\bar{g}) U(\bar{f})
\end{aligned}
$$

so that also the extended representation satisfies the CCR.

However, there is one important point. If $f_{n} \rightarrow \bar{f} \neq 0$, then one knows that $\bar{f}$ defines a linear functional on $\mathscr{V}_{\pi}$ or $\mathscr{V}_{\pi}$ since $\left(f_{n}, g\right)$ converges. But this limit could be zero for all $g$, and $\bar{f}$ would define the null functional. Hence the bilinear form on $\overline{\mathscr{V}}_{\Phi} \times \overline{\mathscr{V}}_{\pi}$ may have become degenerate (cf. the introduction and [1]). The next theorem summarizes the results. 
Theorem 3.2. (Extension Theorem). Let $U(f), V(g)$ be a cyclic representation of the CCR, $f \in \mathscr{V}_{\Phi}, g \in \mathscr{V}_{\pi}$, with cyclic vector $\varphi_{0}$. Then $\mathscr{V}_{\Phi}, \mathscr{V}_{\pi}$ can be uniquely extended to complete metric linear spaces $\overline{\mathscr{V}}_{\Phi}, \overline{\mathscr{V}}_{\pi}$ in the metric $d$ of Theorem 2.6, and the bilinear form $(f, g)$ can be uniquely extended to a (possibly degenerate) bilinear form on $\overline{\mathscr{V}}_{\Phi} \times \overline{\mathscr{V}}_{\pi}$. The representation $U(f), V(g)$ with $f \in \mathscr{V}_{\Phi}, g \in \mathscr{V}_{\pi}$ can be uniquely extended to a unitary representation of the Weyl relations for $\bar{f} \in \overline{\mathscr{V}}_{\Phi}, \bar{g} \in \overline{\mathscr{V}}_{\pi}$.

Corollary 3.3. The function

$$
\bar{d}(\bar{f})^{2}=\int d \lambda e^{-\lambda^{2}}\left\|U(\lambda \bar{f}) \varphi_{0}-\varphi_{0}\right\|^{2}
$$

on $\overline{\mathscr{V}}_{\Phi}$ defines a metric on $\overline{\mathscr{V}}_{\Phi}$ which coincides with $d(f)$ on $\mathscr{V}_{\Phi}$ and is the continuous extension of $d$ from $\mathscr{V}_{\Phi}$ to $\overline{\mathscr{V}}_{\Phi}$.

Proof. Let $f_{n} \rightarrow \bar{f}$ in $\overline{\mathscr{V}}_{\Phi}$. Then $U\left(\lambda f_{n}\right) \rightarrow U(\lambda \bar{f})$ strongly for all $\lambda$. Hence, by Lebesgue's bounded convergence theorem, $d\left(f_{n}\right) \rightarrow \bar{d}(\bar{f})$. The rest of the statement is then obvious. q.e.d.

Note that a similar argument holds for all vectors $\varphi \in \mathfrak{G}$. But it may not be true that

$$
\bar{d}_{1}(\bar{f})=\|U(\bar{f}) \varphi-\varphi\|
$$

defines a metric. This is due to the possible degeneracy of the bilinear form. If $(\bar{f}, g)=0$ for all $g \in \overline{\mathscr{V}}_{\pi}$, then $U(\bar{f})$ may not be a canonical Weyl operator for one degree of freedom, and its spectrum may have discrete points.

If $\bar{f}$ defines the zero functional on $\overline{\mathscr{V}}$, then $U(\lambda \bar{f})$ commutes with all $U$ 's and $V$ 's and hence lies in the center of the von Neumann algebra $\left\{U(f), V(g) ; f \in \mathscr{V}_{\Phi}, g \in \mathscr{V}_{\pi}\right\}^{\prime \prime}$.

Corollary 3.3 means that if one tries to extend the extended representation once more by means of the metric $\bar{d}$ induced by the extended representation, one does not obtain anything new, since $\bar{d}$ coincides with the extension by continuity of $d$ to $\overline{\mathscr{V}}_{\Phi}, \overline{\mathscr{V}}_{\pi}$. Thus $\overline{\mathscr{V}}_{\Phi}, \overline{\mathscr{V}}_{\pi}$ are already complete for $\bar{d}$.

\section{Topological Aspects}

The preceding results, especially Sections 1 and 2, have some immediate topological implications. The metric $d$ of Theorem 2.6 defines a topology $\tau_{\Phi}$ on $\mathscr{V}_{\Phi}$ and $\tau_{\pi}$ on $\mathscr{V}_{\pi}$. If the space does not matter we shall simply write $\tau$. Recall that a topology on a linear space $X$ is a vector topology if 1) $X$ is a Hausdorff space, 2) the mapping $(x, y) \rightarrow x+y$ of $X \times X$ into $X$ is continuous, and if 3) the mapping $(\alpha, x) \rightarrow \alpha x$ of $R_{1} \times X$ into $X$ is continuous. Lemmas 2.1 and 2.5 imply:

Corollary 4.1. $\tau$ is a vector topology. 
Denote by $\tau_{\lambda, \Phi}$ the topology belonging to the metric $d_{\lambda}(f)$ $=\left\|U(\lambda f) \varphi_{0}-\varphi_{0}\right\|$ on $\mathscr{V}_{\Phi}$, and similarly $\tau_{\lambda, \pi}$, where $\varphi_{0}$ is the same vector as used in the construction of the metric $d$. Again we mostly suppress the index $\Phi$ or $\pi$. We further consider the topologies for weak convergence on $\mathscr{V}_{\Phi}, \mathscr{V}_{\pi}$, the so-called weak dual topologies $w\left(\mathscr{V}_{\Phi}, \mathscr{V}_{\pi}\right)$ and $w\left(\mathscr{V}_{\pi}, \dot{\mathscr{V}}_{\Phi}\right)$ on $\mathscr{V}_{\Phi}$ and $\mathscr{V}_{\pi}$, respectively.

A topology $\tau^{\prime}$ is called stronger than a topology $\tau^{\prime \prime}, \tau^{\prime} \geqq \tau^{\prime \prime}$, if every open set of $\tau^{\prime \prime}$ is also open for $\tau^{\prime}$ (i.e., $\tau^{\prime}$ has more (or equal) open sets than $\left.\tau^{\prime \prime}\right)$. Now the results of Section 2 imply the following.

Corollary 4.2. $\tau_{\Phi} \geqq \tau_{\lambda, \Phi} \geqq w\left(\mathscr{V}_{\Phi}, \mathscr{V}_{\pi}\right)$, and $\tau_{\pi} \geqq \tau_{\lambda, \pi} \geqq w\left(\mathscr{V}_{\pi}, \mathscr{V}_{\Phi}\right)$.

Proof. $f_{n} \rightarrow f_{0}(d)$ implies $f_{n} \rightarrow f_{0}\left(d_{\lambda}\right)$, by Lemma 2.2, and $f_{n} \rightarrow f_{0}\left(d_{\lambda}\right)$ implies $f_{n} \rightarrow f_{0}\left(w\left(\mathscr{V}_{\Phi}, \mathscr{V}_{\pi}\right)\right)$. Hence the identity map $\varrho_{0}: f \rightarrow f$ is continuous for the respective topologies [6]. Hence, if $A$ is $\tau_{\lambda}$-open, then $\varrho_{0}^{-1}(A)=A$ is $\tau_{\Phi}$-open, and similarly for $w\left(\mathscr{V}_{\Phi}, \mathscr{V}_{\pi}\right)$. For $\mathscr{V}_{\pi}$ the same applies. q.e.d.

We note in passing that Lemmas 4.1 and 4.2 remain true for any nonzero $\varphi_{0} \in \mathfrak{H}$. For the following, however, it is important that $\varphi_{0}$ be cyclic.

The next theorem, the main result of this section, shows that the topology $\tau$ induced by the metric $d$ is just the topology looked for by Woods [1].

Theorem 4.3. Let $U(f), V(g)$ be a cyclic representation of the CCR in some Hilbert space $\mathfrak{H}$. Let $\tau_{\Phi}$ be the topology on $\mathscr{V}_{\Phi}$ belonging to the metric

$$
d(f)=\left\{\int d \lambda e^{-\lambda^{2}}\left\|U(\lambda f) \varphi_{0}-\varphi_{0}\right\|^{2}\right\}^{\frac{1}{2}},
$$

and let $\tau_{\pi}$ on $\mathscr{V}_{\pi}$ belong to the metric

$$
d(g)=\left\{\int d \lambda e^{-\lambda^{2}}\left\|V(\lambda g) \varphi_{0}-\varphi_{0}\right\|^{2}\right\}^{\frac{1}{2}}
$$

where $\varphi_{0}$ is some arbitrary cyclic vector. Then $\tau_{\Phi}$ is the weakest vector topology on $\mathscr{V}_{\Phi}$ for which $f \rightarrow U(f)$ is strongly continuous, and $\tau_{\pi}$ is the weakest vector topology on $\mathscr{V}_{\pi}$ for which $g \rightarrow V(g)$ is strongly continuous ${ }^{2}$.

Proof. By Theorem 2.6, $f \rightarrow U(f)$ is strongly continuous for $\tau_{\Phi}$ since again sequential continuity suffices. Now let $\tau^{\prime}$ be any vector topology on $\mathscr{V}_{\Phi}$ for which $f \rightarrow U(f)$ is strongly continuous. It will be shown that $\tau^{\prime} \geqq \tau_{\Phi}$.

This is the case if every net [7] $\left\{f_{\alpha}\right\}$ in $\mathscr{V}_{\Phi}$ which converges to 0 in $\tau^{\prime}$ converges to 0 in $\tau_{\Phi}$ also. For this it suffices that $\left\|U\left(\lambda f_{\alpha}\right) \varphi_{0}-\varphi_{0}\right\| \rightarrow 0$ uniformly in $\lambda$ for every bounded interval since then obviously $d\left(f_{\alpha}\right) \rightarrow 0$.

${ }^{2}$ After the completion of this work, we learned that, for the special case of a separable Hilbert space, Araki and Woods have proved the related result that the weakest vector topology is metrizable (H. Araki and E. J. Woods, to be published). 
Assume the convergence to be nonuniform in some interval $I$. Then there exists an $e>0$ such that for each $\alpha$ there exists an $\alpha^{\prime} \geqq \alpha$ and some $\lambda_{\alpha^{\prime}} \in I$ satisfying $\left\|U\left(\lambda_{\alpha^{\prime}} f_{\alpha^{\prime}}\right) \varphi_{0}-\varphi_{0}\right\| \geqq e$. The set of $\lambda_{\alpha^{\prime}}$ forms a net and the $f_{\alpha^{\prime}}$ form a subnet of $\left\{f_{\alpha}\right\}$. There exists a subnet $\left\{\lambda_{\alpha^{\prime \prime}}\right\}$ which converges to some $\lambda_{0} \in I$. The corresponding subnet $\left\{f_{\alpha^{\prime \prime}}\right\}$ converges to 0 . Hence $\lambda_{\alpha^{\prime \prime}} f_{\alpha^{\prime \prime}} \rightarrow 0\left(\tau^{\prime}\right)$ since $\tau^{\prime}$ is a vector topology. Because of strong continuity one then must have $\left\|U\left(\lambda_{\alpha^{\prime \prime}} f_{\alpha^{\prime \prime}}\right) \varphi_{0}-\varphi_{0}\right\| \rightarrow 0$. But $\left\|U\left(\lambda_{\alpha^{\prime \prime}} f_{\alpha^{\prime \prime}}\right) \varphi_{0}-\varphi_{0}\right\| \geqq e$. Hence the assumption is false ${ }^{3}$. The treatment for $\tau_{\pi}$ is identical. q.e.d.

As an immediate consequence of Corollary 2.3 one has the following.

Corollary 4.4. $\tau_{\Phi}$ coincides with the topology of uniform convergence on every bounded interval of $\lambda$ 's of $\left\|U(\lambda f) \varphi_{0}-\varphi_{0}\right\|$.

We note that by the above theorem a different choice of the cyclic vector $\varphi_{0}$ leads to the same topology and hence to an equivalent metric. Since the metric is translation invariant the completion $\overline{\mathscr{V}}_{\Phi}$ depends only on the topology so that the extension of the representation does not depend on the particular $\varphi_{0}$. In the next section it will also be shown that it does not depend on the particular weight function $e^{-\lambda^{2}}$ in the integral for $d$.

Corollary 1.7 shows that for representations of the CCR which are a countable direct sum of cyclic representations there exists a vector $\varphi_{0}$ such that all results derived for cyclic representations remain valid.

\section{Measure Theoretic Aspects}

In this section we first investigate the role of the weight function in the integral (2.2) of the metric $d$ and then discuss some of the results of Section 1 in the framework of the measure theoretic realizations of the CCR by means of a direct integral.

The next result shows that one can take any non-negative function which does not vanish almost everywhere as a weight function and still have the results of Section 2. Also the power 2 in the construction of $d$ is not essential.

Lemma 5.1. Let $U(f), V(g)$ be a representation of the CCR in $\mathfrak{H}$ with $f \in \mathscr{V}_{\Phi}, g \in \mathscr{V}_{\pi}$, and let $\varphi \in \mathfrak{H}, \varphi \neq 0$. Define

$$
d_{\sigma, p}(f)=\left\{\int d \sigma(\lambda)\|U(\lambda f) \varphi-\varphi\|^{p}\right\}^{1 / p},
$$

${ }^{3}$ One can avoid the use of nets by noting that $\left\{(\lambda, f):\left\|U(\lambda f) \varphi_{0}-\varphi_{0}\right\|<\varepsilon\right\}$ is a $\tau^{\prime}$-open set in $R_{1} \times \mathscr{V}_{\Phi}$ and that for each $\lambda$ it contains an open set of the form $U_{\lambda} \times U_{\tau^{\prime}}$ with $(\lambda, 0) \in U_{\lambda}$ and where $U_{\lambda}$ and $U_{\tau^{\prime}}$ are open. By choosing a finite subcovering of $|\lambda| \leqq 1$ it is then easy to find a $\tau^{\prime}$-open set contained in $\left\{f: \sup _{|\lambda| \leqq 1}\left\|U(\lambda f) \varphi_{0}-\varphi_{0}\right\|<\varepsilon\right\}$. The latter sets just form a base of the topology of uniform convergence of $d_{\lambda}(f)$ in $(-1,1)$. 
where $p \geqq 1$ and where $\sigma$ is a finite positive measure on the Borel sets of the real line with nonzero absolutely continuous part (in particular $d \sigma(\lambda)$ $=\varrho(\lambda) d \lambda$ with $\varrho(\lambda) \geqq 0)$. Then $d_{\sigma, p}(f)$ defines a metric on $\mathscr{V}_{\Phi}$, and one has $d_{\sigma, p}\left(f_{n}\right) \rightarrow 0$ if and only if $d_{\lambda}\left(f_{n}\right) \rightarrow 0$ for each real $\lambda$.

Proof. A similar argument as in the proof of Lemma 2.1 shows that $d_{\sigma, p}(f)$ is a metric for an arbitrary measure. Now, if $d_{\lambda}\left(f_{n}\right) \rightarrow 0$ for every $\lambda$, then also $d_{\sigma, p}\left(f_{n}\right) \rightarrow 0$, by Lebesgue's bounded convergence theorem. To show the converse, choose a fixed $\lambda_{0}$ and assume $d_{\sigma, p}\left(f_{n}\right) \rightarrow 0$. Since $\left\|U\left(\lambda_{0} f_{n}\right) \varphi-\varphi\right\|$ is bounded, it suffices to show that every convergent subsequence $\left\|U\left(\lambda_{0} f_{n_{v}}\right) \varphi-\varphi\right\|$ converges to zero. Since the integrand in (5.1) is non-negative, there exists a subsubsequence $\left\|U\left(\lambda f_{n_{v_{K}}}\right) \varphi-\varphi\right\| \rightarrow 0$ $\sigma$-almost everywhere, hence for all $\lambda$ in some set $\Delta$ of positive Lebesgue measure. If $\lambda_{1}, \lambda_{2} \in \Delta$ it then follows by the triangle inequality that also $\left\|U\left(\left(\lambda_{1} \pm \lambda_{2}\right) f\right) \varphi-\varphi\right\| \rightarrow 0$. But the difference set of a set of positive Lebesgue measure contains a finite interval around zero [8], and by finite sums one covers the whole line. Hence $\left\|U\left(\lambda_{0} f_{n_{v}}\right) \varphi-\varphi\right\| \rightarrow 0$. q.e.d.

Lemma 2.2 and the discussion of Section 4 show that the metrics in Eq. (5.1) are all topologically equivalent for all allowed choices of $\sigma$ and $p$. It should be remarked that a purely discrete measure will in general not do. For then there are at most countably many points contributing to the integral, and Woods has shown that strong $\lim _{n} U\left(\lambda_{i} f_{n}\right)=\mathbf{1}$ for countably many $\lambda_{i}$ does in general not imply that $U\left(\lambda f_{n}\right) \rightarrow \mathbf{1}$ for all $\lambda$ (cf. [1]). Together with Corollaries 1.6 and 1.7 this shows the inadequacy of discrete measures.

Theorem 1.5 can be sharpened somewhat for representations of the CCR which allow a realization by means of quasi-invariant measures. Generalizing results of Araki [9] it has been shown in [10] that if the Hilbert space $\mathfrak{H}$ decomposes into a countable direct sum of subspaces which are cyclic for $U(f), f \in \mathscr{V}_{\Phi}$, (as in Lemma 1.3), the representation can be realized as follows. There exists a normed measure $\mu$ on the Borel sets of the space $\mathscr{V}_{\Phi}^{\prime}$ of (algebraically) all linear functionals on $\mathscr{V}_{\Phi} . \mathscr{V}_{\pi}$ can be regarded as a subspace of $\mathscr{V}_{\Phi}^{\prime}$, and $\mu$ is $\mathscr{V}_{\pi}$-quasi-invariant in the sense that if $\mathfrak{N}$ is a null set then so is the translated set $\mathfrak{N}+g$ for each $g \in \mathscr{V}_{\pi}, \mathfrak{S}$ can then be written as a direct integral,

$$
\mathfrak{H}=\int_{\mathscr{V}^{\phi}}^{\oplus} \mathfrak{H}(F) d \mu(F),
$$

$F \in \mathscr{V}_{\Phi}^{\prime}$. The action of $U(f)$ is given by multiplication by $\exp \{i(f, F)\}$. The precise form of $V(g)$ is not of interest here; it has a resemblance to that in the Schrödinger representation.

23 Commun. math. Phys., Vol. 16 
Before proving the sharpened version of Theorem 1.5 we need the following.

Lemma 5.2. Let $\left\{s_{n}\right\}$ be a sequence of real numbers, and let $X \subset R_{1}$ be a set of positive Lebesgue measure. If $e^{i s_{n}}$ does not converge to 1 , then there is an uncountable set $\Lambda \subset X$ of real numbers such that a) $e^{i \lambda s_{n}}$ does not converge to 1 for $\lambda \in \Lambda$, and b) $e^{i\left(\lambda_{1}-\lambda_{2}\right) s_{n}}$ does not converge to 1 for $\lambda_{1}, \lambda_{2} \in \Lambda, \lambda_{1} \neq \lambda_{2}$.

Proof. The set of $\lambda$ 's for which $\left|e^{i \lambda s_{n}}-1\right| \rightarrow 0$ is a measurable set [11], $N$ say. This must be a Lebesgue null set because otherwise, by the same argument as in the proof of Lemma 5.1, $e^{i \lambda_{n}} \rightarrow 1$ for all $\lambda$. Note that $N$ contains with two elements also their sum and difference. The complement $C$ of $N$ has positive measure. Consider the set $C / N$ of all classes $\bar{\lambda}$ of elements of $C$ which differ by some element of $N$, i.e., $\bar{\lambda}=\lambda+N$, $\lambda \in C$. Note that $\lambda+N$ is indeed in $C$. If the number of classes $\bar{\lambda}$ in which elements of $X$ lie were countable, one could choose representatives $\lambda_{1}, \lambda_{2}, \ldots$, such that $X \subset \bigcup_{n}\left(\lambda_{n}+N\right)$, and thus $X$ would be a null set. Hence there are uncountably many classes $\bar{\lambda}_{\alpha}$ of this kind, with representatives $\lambda_{\alpha} \in X$. These fulfill a) and b). q.e.d.

Now we can prove the following theorem which establishes a connection between weak convergence and quasi-invariance.

Theorem 5.3. Let $U(f), V(g)$ be a representation of the CCR in a Hilbert space $\mathfrak{H}$ with $f \in \mathscr{V}_{\Phi}, g \in \mathscr{V}_{\pi}$. Let $\mathfrak{H}$ decompose into a countable direct sum of subspaces cyclic for $U(f), f \in \mathscr{V}_{\Phi}$. Denote by $\mathscr{V}_{\Phi}^{\prime}$ the algebraic dual of $\mathscr{V}_{\Phi}$. Let $\mu$ be the (up to equivalence unique) measure for the direct integral realization. Let $\left\{f_{n}\right\}$ be some sequence in $\mathscr{V}_{\Phi}$, and let $\varphi \in \mathfrak{H}$ be a nonzero vector such that $\left\|U\left(f_{n}\right) \varphi-\varphi\right\| \rightarrow 0$. Let $F^{\prime}$ be some element of $\mathscr{V}_{\Phi}^{\prime}$ such that $\mu$ is quasi-invariant under $\alpha F^{\prime}$ for all $\alpha$ in some set $A$ of positive Lebesgue measure on the real line. Then $\left(f_{n}, F^{\prime}\right) \rightarrow 0$.

Proof. We first show that $e^{i\left(f_{n}, F^{\prime}\right)} \rightarrow 1$. For this it suffices that every convergent subsequence goes to 1 . So one can assume $e^{i\left(f_{n}, F^{\prime}\right)} \rightarrow a$. Let $\varphi$ correspond to $\varphi(F)$ in the direct integral realization of $\mathfrak{H}$. Then

$$
\left\|U\left(f_{n}\right) \varphi-\varphi\right\|^{2}=\int_{\mathscr{V}_{\Phi}^{\prime}}\left|e^{i\left(f_{n}, F\right)}-1\right|^{2}\|\varphi(F)\|^{2} d \mu(F) .
$$

Hence there exists a subsequence $n_{v}$ for which the integrand converges to zero $\mu$-almost everywhere. Let

$$
\mathfrak{B}=\left\{F \in \mathscr{V}_{\Phi}^{\prime}: \lim _{v \rightarrow \infty}\left|e^{i\left(f_{n_{v}}, F\right)}-1\right|=0\right\} .
$$

This set is measurable [11]. Since the set $\left\{F \in \mathscr{V}_{\Phi}^{\prime}:\|\varphi(F)\|>0\right\}$ has positive measure, $\mathfrak{B}$ must have positive measure. Again one has that if $F_{1}, F_{2} \in \mathfrak{B}$ then also $-F_{1}$ and $\left(F_{1} \pm F_{2}\right) \in \mathfrak{B}$. Hence, if $\mathfrak{B}^{c}$ denotes the complement of $\mathfrak{B}$ in $\mathscr{V}_{\Phi}^{\prime}$ and if $F \in \mathfrak{B}^{c}$, then $\mathfrak{B} \pm F \subset \mathfrak{B}^{c}$. 
Now assume $a \neq 1$, and let $\Lambda$ be the set of Lemma 5.2, where $s_{n}=\left(f_{n}, F^{\prime}\right)$ and $X=A$. Since $F^{\prime} \in \mathfrak{B}^{c}$, by assumption, one has, for all $\lambda \in \Lambda, \mathfrak{B}-\lambda F^{\prime}\left(\mathfrak{B}^{c}\right.$ and $\left(\mathfrak{B}-\lambda_{1} F^{\prime}\right) \cap\left(\mathfrak{B}-\lambda_{2} F^{\prime}\right)=\emptyset$ for $\lambda_{1}, \lambda_{2} \in \Lambda, \lambda_{1} \neq \lambda_{2}$; for otherwise $\left(\lambda_{1}-\lambda_{2}\right) F^{\prime} \in \mathfrak{B}$ and $e^{i\left(\lambda_{1}-\lambda_{2}\right)\left(f_{n_{v}}, F^{\prime}\right)} \rightarrow 1$. By the quasiinvariance of $\mu$ under $\alpha F^{\prime}, \alpha \in A, \mu(\mathfrak{B})>0$ implies $\mu\left(\mathfrak{B}-\lambda F^{\prime}\right)>0$ for all $\lambda \in \Lambda$. Hence $\mathfrak{B}^{c}$ contains uncountably many disjoint sets of positive measure and hence must have infinite measure. But $\mu\left(\mathscr{V}_{\Phi}^{\prime}\right)=1$. This contradiction shows that $F^{\prime} \in \mathfrak{B}$ and $a=1$. Hence $e^{i\left(f_{n}, F^{\prime}\right)} \rightarrow 1$. Repeating the same argument for $\beta F^{\prime}$ yields $e^{i \beta\left(f_{n}, F^{\prime}\right)} \rightarrow 1$ for each $\beta$. But then $\left(f_{n}, F^{\prime}\right) \rightarrow 0$, by Eq. (1.2). q.e.d.

Since each $g \in \mathscr{V}_{\pi}$ satisfies the conditions imposed on $F^{\prime}$ in the preceding theorem, one obtains Theorem 1.5 as a corollary for all representations whose Hilbert space is a countable direct sum of subspaces cyclic for $U(f)$, in particular for all representations in a separable Hilbert space. Note, however, that Theorem 1.5 makes no assumptions on the Hilbert space.

An immediate consequence of the above proof is the following.

Corollary 5.4. Let the representation be as in Theorem 5.3. Let $U\left(\lambda f_{n}\right) \rightarrow \mathbf{1}$ strongly for each $\lambda$, and let $\mu$ be quasi-invariant under some $F^{\prime} \in \mathscr{V}_{\Phi}^{\prime}$. Then $\left(f_{n}, F^{\prime}\right) \rightarrow 0$.

Proof. Let $\varphi \in \mathfrak{G}$ be such that $\|\varphi(F)\|=1$ for each $F$. Then

$$
\int d \mu(F)\left|e^{i \lambda\left(f_{n}, F\right)}-1\right|^{2}=\left\|U\left(\lambda f_{n}\right) \varphi-\varphi\right\|^{2} \rightarrow 0 .
$$

Let $\lambda$ be fixed. Again considering convergent subsequences one can assume $e^{i \lambda\left(f_{n}, F^{\prime}\right)} \rightarrow a$. By Eq. (5.4) there exists a subsequence $f_{n_{v}}$ such that $e^{i \lambda\left(f_{n_{v}}, F\right)} \rightarrow 1$ for almost all $F$, i.e., the set $\mathfrak{B}$ of the previous proof satisfies $\mu(\mathfrak{B})=1$. If $F^{\prime} \notin \mathfrak{B}$, then $\mathfrak{B}-F^{\prime} \subset \mathfrak{B}^{c}$, and hence $\mu\left(\mathfrak{B}-F^{\prime}\right)=0$. But then, by the quasi-invariance of $\mu$ under $F^{\prime}, \mu\left(\left(\mathfrak{B}-F^{\prime}\right)+F^{\prime}\right)=\mu(\mathfrak{B})=0$. Hence $e^{i \lambda\left(f_{n}, F^{\prime}\right)} \rightarrow 1$. This holds for each $\lambda$, and hence $\left(f_{n}, F^{\prime}\right) \rightarrow 0$. q.e.d.

In connection with Eq. (5.4) we note that Lemma 1.3 is immediately proved if one chooses $\left\|\varphi_{0}(F)\right\| \equiv 1$. For Eq. (5.4) implies that

$$
\left\|U\left(f_{n}\right) \varphi-\varphi\right\|^{2}=\int d \mu(F)\left|e^{i\left(f_{n}, F\right)}-1\right|^{2}\|\varphi(F)\|^{2} \rightarrow 0
$$

for each $\varphi \in \mathfrak{H}$.

It is clear that results analogous to Theorem 5.3 and Corollary 5.4 also hold for $\mathscr{V}_{\pi}$ and $\mathscr{V}_{\pi}^{\prime}$ if the realization of the representation is based on $\mathscr{V}_{\pi}$ and $\mathscr{V}_{\pi}^{\prime}$. One only has to interchange $U(f), V(g)$ by $V(-g), U(f)$.

Acknowledgments. The authors would like to thank L. Streit and E. J. Woods for helpful comments. 


\section{References}

1. Woods, E. J.: Topologies for test function spaces for representations of the canonical commutation relation. Commun. Math. Phys.

2. Gelfand, I. M., Vilenkin, N. Y.: Generalized functions, Vol. 4. p. 134. New York: Academic Press 1964.

3. Loève, M.: Probability theory, 2nd Ed. p. 192. New York: Van Nostrand 1960.

4. Bourbaki, N.: Topologie generale, 3rd Ed. (Chap. III, Sect. 3, Prop. 5). Paris: Hermann 1960.

5. - Espaces vectoriels topologiques. (Chap. III, Sect. 3, Theorem 4). Paris: Hermann 1955.

6. Dugandji, J.: Topology. (cf. p. 218, Theorem 6.3). Boston: Allyn and Bacon 1966.

7. Kelley, J. L.: General topology. Chapter 2. New York: Van Nostrand, Inc. 1955.

8. Halmos, P. R.: Measure theory. p. 68. New York: Van Nostrand 1950.

9. Araki, H.: J. Math. Phys. 1, 492 (1960); cf. also J. Lew, Thesis, Princeton University (unpublished).

10. Hegerfeldt, G. C., Melsheimer, O.: Commun. Math. Phys. 12, 304 (1969).

11. Ref. [8], p. 84.

G. C. Hegerfeldt

Institut $\mathrm{f}$. Theoret. Physik d. Universität

34 Göttingen, Bunsenstr. 9

J. R. Klauder

Bell Telephone Laboratories

Murray Hill, New Jersey, USA 